\title{
Bose-Einstein Condensation of Helium and Hydrogen inside Bundles of Carbon Nanotubes
}

\author{
F. Ancilotto ${ }^{1}$, M.M. Calbi ${ }^{2}$, S.M. Gatica ${ }^{3}$ and M.W. Cole ${ }^{3}$ \\ ${ }^{1}$ INFM (UdR Padova and DEMOCRITOS National Simulation Center, \\ Trieste, Italy) and Dipartimento di Fisica "G. Galilei", Università di Padova, \\ via Marzolo 8, I-35131 Padova, Italy. \\ 2 Department of Physics, Southern Illinois University, Carbondale, IL 62901-4401 \\ 3 Department of Physics, Pennsylvania State University, University Park, PA 16802
}

(Dated: October 30, 2018)

\begin{abstract}
Helium atoms or hydrogen molecules are believed to be strongly bound within the interstitial channels (between three carbon nanotubes) within a bundle of many nanotubes. The effects on adsorption of a nonuniform distribution of tubes are evaluated. The energy of a single particle state is the sum of a discrete transverse energy $E_{t}$ (that depends on the radii of neighboring tubes) and a quasicontinuous energy $E_{z}$ of relatively free motion parallel to the axis of the tubes. At low temperature, the particles occupy the lowest energy states, the focus of this study. The transverse energy attains a global minimum value $\left(E_{t}=E_{\min }\right)$ for radii near $R_{\min }=9.95 \AA$ for $\mathrm{H}_{2}$ and 8.48 $\AA$ for ${ }^{4} \mathrm{He}$. The density of states $N(E)$ near the lowest energy is found to vary linearly above this threshold value, i.e. $N(E)$ is proportional to $\left(E-E_{m i n}\right)$. As a result, there occurs a Bose-Einstein condensation of the molecules into the channel with the lowest transverse energy. The transition is characterized approximately as that of a four dimensional gas, neglecting the interactions between the adsorbed particles. The phenomenon is observable, in principle, from a singular heat capacity. The existence of this transition depends on the sample having a relatively broad distribution of radii values that include some near $R_{\min }$.
\end{abstract}




\section{INTRODUCTION}

One of the most persistently interesting topics in condensed matter physics is BoseEinstein condensation (BEC). First postulated [1] on the basis of an idealized model (noninteracting particles), BEC was subsequently argued by London to provide an explanation of superfluidity in ${ }^{4} \mathrm{He}$, which is a system consisting of relatively strongly interacting atoms 2]. The London hypothesis has been confirmed in both detailed calculations and careful neutron scattering experiments [3, 4]. More recently, BEC has been observed to occur in ultracold gases consisting of alkali or $\mathrm{H}$ atoms and in systems of excitons at low temperature $T[5,6,7]$.

A logical candidate for the occurrence of $\mathrm{BEC}$ is para- $\mathrm{H}_{2}$, which is the boson equilibrium form of hydrogen at low $T$. The apparent nonexistence of BEC for bulk $\mathrm{H}_{2}$ is attributed to its crystallization below $14 \mathrm{~K}$, which preempts BEC. Some three decades ago, Ginzburg and Sobyanin proposed a search for BEC and superfluidity of $\mathrm{H}_{2}$ in confining geometries (for which crystallization occurs at a lower temperature, if at all) [8]. This suggestion stimulated a number of searches involving both theory and experiments [9, 10, 11, 12, 13, 14, 15, 16]. There has been at least one assertion of successful observation of BEC, involving the lack of damping of rotational motion of an impurity surrounded by hydrogen fluid within a small cluster [9, 14].

In this paper, we describe a different geometry that is predicted to allow BEC to occur 17, 18]. This host material is an ensemble of carbon nanotube bundles. A crucial role is played in this phenomenon by the presence of nonuniformity. In particular, we assume that there exists a fairly broad distribution of nanotube radii present in the experimental sample. We focus our calculations on the spectrum of particles (either $\mathrm{H}_{2}$ or ${ }^{4} \mathrm{He}$ ) confined within interstitial channels (ICs) formed by groups of three nanotubes. It has been argued that both of these species, if allowed access to such channels, are more strongly bound there than on the surface of graphite, the most strongly physisorbing planar surface [19]. There is ambiguous experimental evidence concerning the relative binding energy of these species in the IC and in the grooves on the outside surface of the bundle [20, 21, 22, 23, 24]. We interpret the very large isotope effect seen in isosteric heat data of Wilson et al 22] to indicate particle localization within the ICs for their sample. The specific behavior is evidently sample- dependent, just as our calculations are very sensitive to the assumptions 
that we make.

Much recent research has been devoted to hydrogen within nanotubes bundles, partly due to the exciting prospects of technologies related to hydrogen storage and isotope separation 25, 26, 27, 28, 29]. The vast majority of the theoretical research to date (including that of our group) has assumed a monodisperse distribution of tubes. The present study was stimulated by recent results of Shi and Johnson [30], who showed that the distribution of tube sizes present in actual samples leads to predictions that are quite different from those based on the monodisperse models, in better agreement with experimental data than the naive models' predictions.

The outline of this paper is the following. Section II describes our calculations of the energy spectra of ${ }^{4} \mathrm{He}$ and $\mathrm{H}_{2}$ gases within ICs. Section III employs these results to compute a set of predictions related to BEC that are testable in principle. Section IV presents a further discussion of the model and draws conclusions.

\section{ENERGY SPECTRA AND DENSITY OF STATES OF ADSORBED GASES}

We focus here on those states having the lowest energy since they are the most important for the BEC phenomenon; higher energy behavior can be explored with the same approach but minor changes in the analysis are required. The energy of a particle confined within an $\mathrm{IC}$ is evaluated with the Schrodinger equation. Assuming that the particular IC is perfectly straight, this equation is separable; then the total energy is given by the relation:

$$
\begin{gathered}
E_{n}(p)=E_{n}^{\text {trans }}+E^{\text {long }}(p) \\
E^{\text {long }}(p)=\frac{p^{2}}{2 m}
\end{gathered}
$$

Here, the first term is the $n$-th eigenvalue of the Schrodinger equation describing motion transverse to the axis of the IC and the second term is the quasicontinuous kinetic energy of motion parallel to the axis. We assume for simplicity that the longitudinal kinetic energy is determined by the free particle mass; if the potential were corrugated the band mass should be used instead of $m$ to characterize the low energy states [31, 32]. Such a substitution would lead to a straightforward change in the numerical and analytical results below. 
In the present circumstance, the transverse eigenvalues are separated by energies (of order $100 \mathrm{~K}$ ) that are much larger than relevant temperature scales [33]. Hence, we need consider only the lowest eigenvalue $E_{1}^{\text {trans }}$ of transverse motion. We denote this quantity $E_{t}(\mathbf{R})$, where the vector $\mathbf{R}=\left(R_{1}, R_{2}, R_{3}\right)$ has components equal to the radii of the tubes surrounding the IC. We refer to the domain of possible ICs as $\mathbf{R}$ space; a given IC is represented by one point in this space while a sample with many ICs is described by a cloud of points in this space. The single particle spectrum is now specified by the relation

$$
E(\mathbf{R}, p)=E_{t}(\mathbf{R})+\frac{p^{2}}{2 m}
$$

Here, $E_{t}(\mathbf{R})$ is computed following the procedure of Stan et.al. 34]. To get a sufficiently accurate dependence of $E_{t}$ on $\mathbf{R}$, one must include anisotropic and anharmonic contributions to the potential in the IC. We note that alternative parametrizations of the potential have been proposed. Table 1 presents one such alternative result for $E_{t}$. The qualitative behavior presented in the remainder of this paper is the same for the case of that alternative potential.

Of considerable interest is the density of states $h(E, \mathbf{R})$ for a single IC. For a specific set of adjacent tubes $(\mathbf{R})$, this function is given by a sum over all momenta, which may be replaced by an integral:

$$
h(E, \mathbf{R})=\sum_{p} \delta[E-E(\mathbf{R}, p)]=\frac{L}{\pi \hbar}\left(\frac{m}{2}\right)^{1 / 2} \frac{1}{\left(E-E_{t}(\mathbf{R})\right)^{1 / 2}} \Theta\left[E-E_{t}(\mathbf{R})\right]
$$

Here $L$ is the length of the channel and the usual procedure for quantizing the 1D motion has been followed. The inverse square root dependence on the energy above threshold is characteristic of 1D motion. The last factor involves the Heaviside step function $\Theta(x)$, which is unity here for $E>E_{t}(\mathbf{R})$ and zero otherwise.

Now let us consider an ensemble of nanotubes. The lowest energy states (those dominating the low temperature behavior) are concentrated near the minimum of the function $E_{t}(\mathbf{R})$. As might be expected, the global minimum of $E_{t}(\mathbf{R})$ is found for the symmetric case, $\left\{R_{i}\right\}=R_{\text {min }}$. We define the vector specifying this IC as $\mathbf{R}_{\min }=R_{\min }(1,1,1)$ and $E_{\min }$ as the corresponding energy. The numerical results for these quantities appear in Table 1.

The behavior of the function $E_{t}(\mathbf{R})$ near this minimum is remarkable. If one considers only symmetric ICs (bounded by tubes of identical radii), the energy varies rapidly as a function of the difference $\left|\mathbf{R}-\mathbf{R}_{\text {min }}\right|$. However, the spectrum of the very lowest energy 
states is dominated by asymmetric ICs for which one of the radii equals $R_{\min }$ and the two others differ from $R_{\text {min }}$ by equal, but opposite amounts, e.g. $\mathbf{R}=R_{\min }(1,1+x, 1-x)$, where $x \ll 1$. The energy varies extremely slowly with $x$ in such a case. This is depicted for $\mathrm{H}_{2}$ in Fig. 1, which shows the energy variation in a plane within $\mathbf{R}$ space that contains both this variable $x$ line and the diagonal $(1,1,1)$ line. (Analogous behavior occurs for the case of ${ }^{4} \mathrm{He}$ ). One observes in the figure a very narrow valley of low-lying states of variable $x$, along the $(0,1,-1)$ direction, where the origin is shifted to $\mathbf{R}_{\text {min }}$. Similar small gradient behavior of $E_{t}(\mathbf{R})$ is found along equivalent permutations of this direction. Note that the higher energy contours are extended, nearly straight lines perpendicular to the diagonal. These latter contours imply an energy dependence of the density of states that is qualitatively different from that based on contours of lower energy (near the valleys).

We next evaluate the transverse density of states for a given experimental sample of tubes. We denote by $\nu(\mathbf{R})$ the density distribution of ICs, defined so that $\nu(\mathbf{R}) d \mathbf{R}$ is the number of ICs present in the sample within a volume $d \mathbf{R}$ in $\mathbf{R}$ space. In this paper, we focus on low energy behavior corresponding to such close proximity to $\mathbf{R}_{\min }$ that we may replace $\nu(\mathbf{R})$ by $\nu\left(\mathbf{R}_{\text {min }}\right)$; future studies will address more general situations for which that approximation is not adequate. In the following discussion, we assume that $\nu(\mathbf{R})$ is a sufficiently broad distribution that ICs near this minimum exist in sufficient numbers to treat adsorption statistically. Moreover, we assume that the set of tube sizes forms a continuum. The former assumption is well justified in experimental samples produced to date involving large numbers of tubes, which typically have a dispersion in radius values of order $20 \%$ [35, 36]. The latter (continuum) assumption is justified by the following argument. Near radius $1 \mathrm{~nm}$, there are many values of the chiral indices that yield quite similar radii; the mean spacing between successive values of the radius is quite small, $0.002 \mathrm{~nm}$. In addition, the ICs experience small perturbations due to their environment; in particular, the ICs at the center of a bundle are expected to be compressed relative to those near the perimeter. A model calculation (unpublished) yields an expression for the difference $\delta$ between the tubetube separation in an infinite rope and that $\left(R_{1}\right)$ of an isolated nanotube pair; apart from a constant of order one, the result is $\delta=\left[C \lambda^{2}\right] /\left[k R_{1}^{6}\right]$, where $C$ is the van der Waals-London intercarbon interaction coefficient $\left(C \sim 20 \mathrm{eV}-\AA^{6}\right), \lambda$ is the $1 \mathrm{D}$ density of carbon atoms in a nanotube $\left(\lambda \sim 15 \AA^{-1}\right), R_{1} \sim 17 \AA$ is the spacing between tube centers and $k=10^{-3} \mathrm{eV} / \AA^{3}$ is the force constant per unit length associated with neighboring tubes' interactions [37]. 
The result, $\delta \sim 0.15 \AA$, represents a nearly $5 \%$ compression of the lattice constant of ropium relative to the separation of an isolated pair. This shift in separation is consistent with estimates of the analogous shift in breathing mode frequency [38]. On the basis of these arguments, the assumption of a continuum of possible radii appears appropriate.

The transverse density of states $g(E)$ for a given sample of tubes is expressed by the relation

$$
g(E)=\int d \mathbf{R} \nu(\mathbf{R}) \delta\left[E-E_{t}(\mathbf{R})\right]
$$

The total density of states $N(E)$ is found by summing over all states of the ICs present in the sample:

$$
\begin{gathered}
N(E)=\int d \mathbf{R} \sum_{p} \delta[E-E(\mathbf{R}, p)] \nu(\mathbf{R}) \\
N(E)=\frac{L}{\pi \hbar}\left(\frac{m}{2}\right)^{1 / 2} \int_{E_{\text {min }}}^{E} d E_{t} g\left(E_{t}\right)\left[E-E_{t}\right]^{-1 / 2}
\end{gathered}
$$

Note that in the special case of a perfectly uniform distribution of $N_{I C}$ identical ICs, $\nu(\mathbf{R})=$ $N_{I C} \delta\left(\mathbf{R}-\mathbf{R}_{0}\right)$, yielding $g(E)=N_{I C} \delta\left[E-E_{t}\left(\mathbf{R}_{0}\right)\right]$; then $N(E)$ is precisely $N_{I C}$ times the single tubes density of states, $h\left(E, \mathbf{R}_{0}\right)$, given in Eq. 4 above, as expected.

Figs. 2 and 3 present the transverse densities of states $g\left(E_{t}\right)$ for $\mathrm{He}$ and $\mathrm{H}_{2}$, respectively, computed numerically from a distribution of $600^{3}$ tubes with radius values spread uniformly over the interval $8.5 \AA$ to $11.5 \AA$. In both cases, the behavior of $g(E)$ at low energy is characterized by a power law dependence on energy above threshold : $g_{\text {low }}(E) \sim(E-$ $\left.E_{\text {min }}\right)^{1 / 2}$. At higher energy, there is a decrease of $g(E)$ with energy, fit well by the expression $g_{\text {high }}(E) \sim\left(E-E_{\text {min }}\right)^{-1 / 2}$. The initial behavior is explained by the following argument. At low energy, the density of states is obtained by counting the number of ICs whose energy $E_{t}(\mathbf{R})$ lies in an interval between $E$ and $E+d E$. Assume for simplicity that the isoenergy surfaces are spherical surfaces centered about the point $\mathbf{R}=\mathbf{R}_{\min }$ (i.e. $E-E_{\min }=$ $\left.(k / 2)\left|\mathbf{R}-\mathbf{R}_{\text {min }}\right|^{2}\right)$. Then $g(E)$ is obtained from the number of points in the shell between spherical surfaces (corresponding to $E$ and $E+d E$ ) centered at $\mathbf{R}_{\min }$. The result of this simple model is then 


$$
g_{\text {low }}(E)=\nu\left(\mathbf{R}_{\text {min }}\right) 4 \pi\left(\frac{2}{k^{3}}\right)^{1 / 2}\left(E-E_{\text {min }}\right)^{1 / 2}
$$

This dependence on the square root of the energy turns out to be valid even in the extreme anisotropic case of interest here [39]. The result then is that $k^{3}$ is replaced by $k_{\text {anis }}^{3}=k_{x} k_{t}^{2}$, where $x$ denotes the $(1,1,1)$ direction in $\mathbf{R}$ space and $t$ denotes the two directions transverse to that.

Note that this square root energy dependence of $g_{\text {low }}(E)$ coincides with that of the density of states of a 3D gas. The higher energy behavior $(1 / \sqrt{E})$ of $g_{\text {high }}(E)$ is quite different. The relevant high energy regime in Fig. 1 is that where the energy surfaces in $\mathbf{R}$ space are perpendicular to the $(1,1,1)$ diagonal. Since this variation is essentially a $1 \mathrm{D}$ dependence, the resulting high energy form of $g_{\text {high }}(E)$ is that of a 1D system, as in Eq. 4 above; this gives rise to a behavior $g_{\text {high }}(E) \sim\left(E-E_{\text {min }}\right)^{-1 / 2}$ (as is observed in Figs. 2 and 3). We remark that this energy-dependent variation in effective dimensionality has analogs in electronic band structures, where wave vector replaces the $\mathbf{R}$ variable as the source of the unusual dependence. [40]

Figs. 2 and 3, lower panels, depict the total densities of states $N(E)$ derived from Eq. [7. In view of the power law forms (e.g. Eq. 8) of $g(E)$, we expect power laws for $N(E)$ in appropriate regimes of $E$. For example, the very lowest energy behavior is determined by the integral

$$
\int_{E_{\text {min }}}^{E} d E_{t}\left(E_{t}-E_{\text {min }}\right)^{1 / 2}\left(E-E_{t}\right)^{-1 / 2}=\left(E-E_{\text {min }}\right) \int_{0}^{1} d y[y /(1-y)]^{1 / 2}=\frac{\pi}{4}\left(E-E_{\text {min }}\right)
$$

$$
N(E)=\nu\left(\mathbf{R}_{\text {min }}\right) \frac{L \pi}{\hbar}\left(\frac{m}{k_{\text {anis }}^{3}}\right)^{1 / 2}\left(E-E_{\text {min }}\right)
$$

Note that the prefactor of the energy difference (which we call $a$ ) is proportional to the volume of the nanotubes, so that $N(E) / a$ is an intensive variable. The linear dependence on energy above threshold $\left(E-E_{\min }\right)$ is the behavior characteristic of a $4 \mathrm{D}$ gas. This initially unexpected result is a logical consequence of convoluting a transverse spectrum characteristic of a 3D system (eq. 8) with the 1D longitudinal degree of freedom. Similarly, the behavior at higher energy is obtained by convoluting an inverse square root dependence 
of $g_{\text {high }}(E)$ with the 1D inverse square root dependence. Then the high energy result is that $N(E)$ is independent of energy, behavior characteristic of a $2 \mathrm{D}$ density of states. These analytic results for the spectra are consistent with the numerical results seen in Figs. 2 and 3 ; that is, the low energy density of states is proportional to $\left(E-E_{\min }\right)$ while the high energy behavior is independent of energy. By the word "high", we mean energies reasonably close to $E_{\min }$ (say $E-E_{\min } \approx 20 \mathrm{mK}$ ) but not the absolutely lowest energies.

To summarize this section, we note that unexpected behavior of $N(E)$ emerges from a simple model. The low energy spectrum of the nonuniform system is qualitatively different from the spectrum obtained when heterogeneity is ignored. The latter corresponds to 1D physics, i.e. $N(E)$ proportional to $\left(E-E_{\min }\right)^{-1 / 2}$. With heterogeneity, instead, we find that $N(E)$ exhibits a 4D form at very low energy and a 2D form at higher energy.

\section{PREDICTION OF BEC}

In this section we explore the consequences of the anomalous densities of states for thermal properties of these systems. In so doing, we assume that the adsorbed gas can equilibrate. This entails the rearrangement of the particles as the temperature $\mathrm{T}$ is lowered by moving from ICs that have high transverse energies to those with lower energies. Thus, we assume a combination of sufficient particle mobility and sufficiently patient experimentalists that the assumption is valid. In the extreme opposite limit of negligible transport of particles the thermal behavior is that of isolated and independent $1 \mathrm{D}$ systems. The difference between these behaviors is dramatic.

At very low density, one can ignore the effects of both interparticle interactions and quantum statistics. Then, the specific heat $C(T)=d U / d T$ at fixed number $\left(N_{p}\right)$ of particles can be computed from the classical Boltzmann expression for the mean energy $U$ :

$$
\left(\frac{U(T)}{N_{p}}\right)_{\text {classical }}=\frac{\int d E E N(E) \exp [-\beta E]}{\left.\int d E N(E)\right] \exp [-\beta E]}
$$

Here $\beta^{-1}=k_{B} T$. One can insert possible power law forms $N(E) \sim E^{(d / 2-1)}$ corresponding to a gas in $d$ dimensions and derive the familiar relation

$$
\left(\frac{C}{N_{p} k_{B}}\right)_{\text {classical }}=\frac{d}{2}
$$


Fig. 4 presents the resulting classical behavior for the systems of $\mathrm{He}$ and $\mathrm{H}_{2}$ in nanotubes. One observes that the low $T$ regime does correspond to the expected 4D gas behavior, $C /\left(N_{p} k_{B}\right)=2$ while the high $\mathrm{T}$ regime exhibits $2 \mathrm{D}$ gas behavior, $C /\left(N_{p} k_{B}\right)=1$. The initial rise above 2 at very low $T$ is explained by a small upward deviation from linearity found in $N(E)$ at low $E$. That is, if $N(E)$ at low $E$ is proportional to $E\left(1+E / E_{1}\right)$, where $E_{1}$ is some constant, then $\left[C /\left(N_{p} k_{B}\right)\right]_{\text {classical }}=2\left[1+2 /\left(\beta E_{1}\right)\right]$. The crossover between the $4 \mathrm{D}$ and $2 \mathrm{D}$ regimes is gradual, beginning at a few $\mathrm{mK}$ and complete by $20 \mathrm{mK}$; the latter value corresponds to the energy above which $N(E)$ is virtually identical to the $2 \mathrm{D}$ form.

At higher density, one must consider the effects of interactions and quantum statistics. In the present treatment we ignore interparticle interactions, except for some comments in the next section. While this is a common assumption, usually adopted for simplicity, there exists some justification for it in the present instance because of electrodynamic screening of the van der Waals interaction by the surrounding medium; there are additional elastic screening effects that have yet to be studied [41]. We do not know the resulting effective interaction well enough to characterize it. In contrast, the effects of quantum statistics are easily evaluated by the standard procedures. In particular, the assumed constraint of fixed particle number yields an implicit relation for the chemical potential $\mu$, which depends on $T$, from which one may derive the energy:

$$
\begin{gathered}
N_{p}=\int d E n(E, T) \\
U(T)=\int d E \operatorname{En}(E, T)
\end{gathered}
$$

$$
n(E, T)=N(E)(\exp [\beta(E-\mu)]-1)^{-1}
$$

(As usual, the classical equations 11 and 12 are obtained in the limit when the fugacity $Z=\exp (\beta \mu) \ll 1)$. From $U(T)$, one may compute $C(T)$. In evaluating these formulae, one encounters a phenomenon that is well known to occur in 3D bose gases: BEC. This transition is derived here in the usual way, by evaluating the implication for the variable $\mu$ (at fixed $N_{p}$ ) as $T$ is lowered: upon decreasing $T, \mu$ increases up to the point where it 
reaches its highest possible value $\mu=E_{\text {min }}$. Thus, the BEC condition for the transition temperature $T_{c}=\left(k_{B} \beta_{c}\right)^{-1}$ is

$$
N_{p}=\int d E N(E)\left(\exp \left[\beta_{c}\left(E-E_{\text {min }}\right)\right]-1\right)^{-1}
$$

Since $\mu$ cannot exceed $E_{m i n}$, as $T$ falls below $T_{c}$ (given implicitly by this relation), there ensues a division into two groups of particles- the $N_{\text {excited }}$ particles accounted for by the integral over excited states $\left(E>E_{\text {min }}\right)$ and the remaining particles having $E=E_{\text {min }}$ that comprise the bose condensate, $N_{\text {cond }}$ :

$$
\begin{gathered}
N_{\text {cond }}=N_{p}-N_{\text {excited }}\left(T<T_{c}\right) \\
N_{\text {excited }}=\int d E N(E)\left(\exp \left[\beta\left(E-E_{\text {min }}\right)\right]-1\right)^{-1}
\end{gathered}
$$

The results of these calculations appear in Figs. 5 to 8. Fig. 5 depicts the dependence of $Z$ on the relative temperature $T / T_{c}$ for two different densities. Fig. 6 displays the dependence on $T$ of the condensate fraction $f(T)=N_{\text {cond }} / N_{p}$. Fig. 7 shows the function $C(T)$, which exhibits singular behavior at the transition. Finally, Fig. 8 shows the dependence of $T_{c}$ on $N_{p}$. Note that the transition temperature falls within the $\mathrm{mK}$ range studied in many low temperature laboratories, so experimental observation of this transition is feasible.

In the preceding three figures, comparison is made with results obtained for a purely $4 \mathrm{D}$ bose gas defined to have the same initial slope of the density of states as the real system. That ideal system satisfies $N(E)=a E$, for all $E>0$, with the analysis presented in the Appendix. (For the real system, the $a$ coefficient is the prefactor of the energy term in Eq. 10). The key results for the $4 \mathrm{D}$ system are

$$
\begin{gathered}
f_{4 D}(T)=1-\left(\frac{T}{T_{c, 4 D}}\right)^{2} \\
C_{4 D}(T)=\frac{36 \zeta(3)}{\pi^{2}}\left(\frac{T}{T_{c, 4 D}}\right)^{2}, T<T_{c, 4 D} \\
T_{c, 4 D}=\left(\frac{6 N_{p}}{a}\right)^{1 / 2} \frac{1}{\pi k_{B}}
\end{gathered}
$$


Here $\zeta(3)=1.202$ is a Riemann zeta function. The $T^{2}$ dependences of $f(T)$ and $C(T)$ are exhibited by the "real" system at low $T$, as seen in the figures. At the very lowest temperatures, only the lowest energy states play a role; since $N(E)$ is the same for the ideal system in this region, the behaviors coincide. The transition temperature is higher for the real system than for the ideal one because at high $E, N(E)$ is lower in the real case than in the ideal case, implying that (for the same value of $N_{p}$ ) $\mu$ reaches its transition value $E_{\text {min }}$ at a higher value of $T_{c}$.

Since the ground state energy of the noninteracting classical system coincides with that of the noninteracting quantum system, as does the energy at very high $T$, it follows that the integral of the two heat capacities is the same. At any particular $T$, of course, the values of $C$ are very different. In particular, the quantum system manifests the reduced $C$ at low $T$ expected in this bose gas case. The quantum system exhibits a much larger value of $\mathrm{C}$ in the vicinity of $T_{c}$, which is dominated by a large rate of excitation out of the condensate; see Fig. 7.

One interesting question is this: how are the particles distributed in energy as a function of $T$. To address this, we present in Fig. 9 the occupied energy distribution function $n(E, T)$ at various temperatures. One observes the increasing relative occupation of low energy states with decreasing $T$; for $T>T_{c}$, their sum is just $N_{p}$, the total number present. At $T_{c}$ there occurs a discontinuous jump in the occupation of the lowest energy states accompanying the BEC transition. Below $T_{c}$, the number $N_{\text {excited }}$ of uncondensed particles decreases, vanishing at $T=0$, when all of the particles settle into the condensate.

Our prediction of BEC in this anisotropic system raises a question: what is the relationship between the transition condition and that of more familiar homogeneus problems, e.g. the 3D Bose gas and the Kosterlitz-Thouless transition of a superfluid film? In the latter examples, the relevant criterion is if the form $\rho \lambda^{d} \sim 1$, where $\rho$ is the number density, $d$ the dimensionality and $\lambda=\left(2 \pi \beta \hbar^{2} / m\right)^{1 / 2}$ is the de Broglie thermal wavelength. We present now a heuristic argument that is consistent with this homogeneus relation and yields a good estimate of the transition temperature for the present BEC problem. The argument estimates $T_{c}$ by analyzing the condition for quantum degeneracy; the number of particles $N_{p}$ becomes comparable to the number of states $N_{\text {states }}\left(T_{c}\right)$ lying within $k_{B} T_{c}$ of the ground state. The number of states within that interval is essentially the product of the number of states of longitudinal motion within that range and the number of states of transverse motion within 
that range:

$$
\begin{aligned}
N_{\text {states }}(T) & \sim N_{\|}(T) N_{\perp}(T) \\
& \sim\left(\frac{\left(m k_{B} T\right)^{1 / 2}}{\hbar / L}\right)\left(\frac{\nu}{\left(\beta k_{\text {anis }}\right)^{3 / 2}}\right)
\end{aligned}
$$

The factor $N_{\|}$is the ratio of the thermal momentum in the $z$ direction to the spacing between discrete states. The factor $N_{\perp}$ is derived from a thermal spread of states in $\mathbf{R}$ space $\left[\delta R \sim\left(\beta k_{\text {anis }}\right)^{-1 / 2}\right]$ and the corresponding number of states in the experimental sample, $N_{\perp} \sim \nu \delta R^{3}$. Setting $N_{p}=N_{\text {states }}\left(T_{c}\right)$ yields our estimate of the BEC transition condition:

$$
N_{p} \sim \frac{L \nu}{\hbar \beta^{2}} \frac{m^{1 / 2}}{k_{\text {anis }}^{3 / 2}}
$$

Apart from numerical factors, this result coincides with the combination of Eqs. 10 and 21. Note that this last equation can be rewritten in the form $\rho^{*} \lambda^{4} \sim 1$ with $\rho^{*}=N_{p} /\left(L l^{3}\right)$ where $l=\nu^{1 / 3} \hbar /\left(m k_{\text {anis }}\right)^{1 / 2}$ is a characteristic length defined by the energetic parameters and the distribution of radii.

\section{SUMMARY AND DISCUSSION}

In this paper we describe a conceptually simple system that turns out to exhibit remarkable behavior. The focus of the study is the lowest-lying states of a system of hydrogen or helium moving within a collection of nanotubes having many ICs. Our analysis yields a density of states $N(E)$ that fulfills the requirement of BEC (i.e. the maximum integrated occupation number is finite). Equally interesting, perhaps, is the finding that the calculated properties at low $T$ are characteristic of a $4 \mathrm{D}$ gas in free space. Since (to the best of our knowledge) no 4D gas has been observed previously, the predicted low $\mathrm{T}$ and critical behaviors of this system are particularly interesting.

A number of theoretical issues need to be discussed. First, one must ask whether the ideal gas assumption is valid; indeed, our group has previously explored the vapor-liquid transition of these gases using quasi-1D models of perfect ICs [42]. A concern is therefore that such condensation in a somewhat disordered system would either preempt the BEC or alter the nature of the BEC, as is the situation with $3 \mathrm{D}$ superfluid ${ }^{4} \mathrm{He}$. However, it may 
well be that disorder reduces the temperature of the hypothetical condensation transition below that of the BEC transition, so that two separate transitions occur.

A related question is the role of screening by the medium of the interparticle interaction. On the bare surface of graphite, experimental and theoretical evidence has indicated that the well-depth is reduced by some 10 to $20 \%$ [43]. Intuition and some calculations [41] suggest that the attraction would be reduced by a much greater factor for gases within a nanotubes array. That is consistent with the neglect of these interactions in the present paper.

The requirements for observation of this phenomenon in the laboratory are not trivial to satisfy. A particularly serious concern is that equilibrium is difficult to achieve. It requires particles to diffuse out of one IC and into another as $T$ changes. The slow equilibration is a potentially fatal problem that is difficult to assess; experimental diffusion data would be very helpful in this regard. [44, 45] We note that one need not imagine that particles are required to move macroscopic distances; one can employ tubes of quite finite length in order to accelerate the process of equilibration. Evidently, one should not underestimate this problem, which is an important concern in much broader contexts involving nanotubes, such as gas storage and isotope separation.

The problem at hand raises the venerable question of the relation between BEC and superfluidity (SF). 46 We know that BEC and SF appear simultaneously at the lambda transition of ${ }^{4} \mathrm{He}[3,4]$. However, the Kosterlitz-Thouless transition of 2D films is one involving SF without BEC [47]. Moreover, the 3D ideal bose gas exhibits BEC without $\mathrm{SF}$, since the Landau velocity criterion is not satisfied. The latter says that the threshold velocity for superflow is the minimum of the ratio of the excitation energy to the momentum [48]. Our unusual nanotubes-hosted BEC state would also fail this test, implying that no superflow is possible. Another aspect of SF is quantized circulation, which is impossible in this environment. Thus, we think that SF is unlikely to accompany the transition described above. If, however, one were to include weak interparticle interactions, SF could occur.

The ideal nanotube radii for the lowest energy states fall in the range 0.8 to $1 \mathrm{~nm}$, which is an advantageous size because typical samples contain many such tubes. If, for example, only smaller tubes are present in a particular sample, one would have to explore the behavior without all of the simplifying mathematics employed here. We have yet to tackle that problem in general, but do note some interesting results found in one case. Suppose for simplicity that the transverse density of states for a given sample (consisting of $\mathrm{N}_{I C}$ ICs) 
has a form (that is quantitatively wrong but qualitatively reasonable in that it consists of a spread of transverse energies) given by the "uniform barrier" function:

$$
\begin{gathered}
g\left(E_{t}\right)=\frac{N_{I C}}{\left(E_{>}-E_{<}\right)}, E_{<}<E_{t}<E_{>} \\
g\left(E_{t}\right)=0, \text { otherwise }
\end{gathered}
$$

The result in this case (from Eq. 71) is

$$
N(E)=\frac{2 N_{I C}}{\left(E_{>}-E_{<}\right)} \frac{L}{\pi \hbar}\left(\frac{m}{2}\right)^{1 / 2}\left(\left(E-E_{<}\right)^{1 / 2} \Theta\left[E-E_{<}\right]-\left(E-E_{>}\right)^{1 / 2} \Theta\left[E-E_{>}\right]\right)
$$

This expression yields the 1D limiting case of Eq. 4 when the barrier width $\left(E_{>}-E_{<}\right)$falls to zero. In the case of nonzero width, this expression yields $3 \mathrm{D}$ behavior for $N(E)$ at low $\mathrm{E}$ and 1D behavior at high $E$. The reason for the 3D limit is that the constant transverse density of states in the finite uniform barrier model mimics the constant density of states of a 2D gas. In this model, one derives a BEC transition from this form of $N(E)$, with thermal behavior quite different from that described elsewhere in this paper, i.e., when the lowest possible transverse energies (near $E_{\text {min }}$ ) play the dominant role in determining the behavior. The transition temperature of a system of $N_{p}$ molecules, obtained for the uniform barrier model with the conventional 3D theory, satisfies

$$
\left(k_{B} T_{c}\right)^{3}=\frac{2 \pi}{m}\left(\frac{N_{p}\left(E_{>}-E_{<}\right) \hbar}{L N_{I C} \zeta(3 / 2)}\right)^{2}
$$

This proportionality of $T_{c}$ to $N_{p}^{2 / 3}$ is characteristic of $3 \mathrm{D}$ behavior, in contrast with the $4 \mathrm{D}$ proportionality to $N_{p}^{1 / 2}$ in Eq. 21.

One might ask whether similar BEC phenomena occur in a related environment, the groove between two nanotubes (e.g. at the external surface of a bundle). Adsorption in this region has been much studied in theory and experiments [4]. We have therefore investigated the consequence of heterogeneity for that problem, with interesting results. One finding is that the symmetric situation (two identical radii) provides a local maximum (in 2D $\mathbf{R}$ space) of the transverse energy. Hence, the methodology and results of the present paper are not applicable, for the most part. The one related result is that there occurs in the groove case an anomalous signature of inhomogeneous broadening in the excitation spectrum. This is 
qualitatively analogous to the behavior of $N(E)$ here, except that spectroscopy is the tool of choice for its investigation. [17]

In closing, we emphasize an intriguing fact: this transition is a consequence of the nonuniformity of the ICs within experimental samples. A perfectly uniform set of ICs would exhibit 1D physical properties instead of the transition described here. Such a remarkable consequence of heterogeneity has precedents in other physical systems, of which the spin glass phenomenon is a well-known example [50, 51, 52].

We are grateful to Carlo Carraro, Vincent Crespi, Allan Griffin, Susana Hernández, Paul Lammert, Ari Mizel, Aldo Migone, Paul Sokol and Flavio Toigo for helpful discussions. This research has been supported by the National Science Foundation. F.A. acknowledges funding from MIUR-COFIN 2001.

\section{APPENDIX: 4D IDEAL BOSE GAS}

We consider an ideal bose gas in four dimensions whose density of states $N(E)$ satisfies

$$
N(E)=a E(E>0)
$$

For this system, we evaluate the number of particles, energy and specific heat in the usual way, using Eqs. 13-15. Straightforward calculations yield

$$
N_{p}=a g_{2}(Z)\left(k_{B} T\right)^{2}+N_{\text {cond }}
$$

where $N_{\text {cond }}$ is the number of bose condensed particles, and

$$
U=2 a g_{3}(Z)\left(k_{B} T\right)^{3}
$$

Here the functions $g_{2}$ and $g_{3}$ are second and third "Bose Einstein integrals",

$$
g_{n}(Z)=\frac{1}{\Gamma(n)} \int_{0}^{\infty} \frac{x^{n-1} d x}{Z^{-1} e^{x}-1} .
$$

The fugacity $Z(T)$ is determine from Eq. A.2. Moreover, from this equation we obtain

the critical temperature $T_{c}$ for a given number of particles $N_{p}$, given that the maximum value of $Z$ is 1 and $g_{2}(Z)$ is a monotonically increasing function, 


$$
k_{B} T_{c}=\sqrt{\frac{N_{p}}{\zeta(2) a}}
$$

From Eqs. A.5 and A.2 the condensate fraction results,

$$
f=\frac{N_{\text {cond }}}{N_{p}}=1-\left(\frac{T}{T_{c}}\right)^{2} .
$$

Now we evaluate the specific heat. To do so we have to be aware that the fugacity depends on the temperature only for $T>T_{c}$ while it is equal to 1 for $T \leq T_{c}$. Then we obtain

$$
\begin{aligned}
\frac{C_{N}}{N_{p} k_{B}} & =6 \frac{g_{3}(Z)}{g_{2}(Z)}-4 \frac{g_{2}(Z)}{g_{1}(Z)}\left(T \geq T_{c}\right) \\
& =6 \frac{\zeta(3)}{\zeta(2)}\left(\frac{T}{T_{c}}\right)^{2}\left(T \leq T_{c}\right)
\end{aligned}
$$

Note that $C_{N}$ is a continuous function of $\mathrm{T}$, with its value at the critical temperature $C_{v}\left(T_{c}\right)=4.4 N_{p} k_{B}$.

In the these derivations we have used some properties of the functions $g_{n}(Z)$,

$$
g_{n}(1)=\zeta(n)
$$

and

$$
Z g_{n}^{\prime}(Z)=g_{n-1}(Z) .
$$

These, together with equation A.2, give the following relationship,

$$
\frac{\partial g_{2}(Z)}{\partial T}=-\frac{2}{T} g_{2}(Z)
$$




\begin{tabular}{|l|c|c|c|c|}
\hline & $\sigma(\AA)$ & $\epsilon(\mathrm{K})$ & $R_{\text {min }}(\AA)$ & $E_{\text {min }}(\mathrm{K})$ \\
\hline \hline $\mathrm{H}_{2}{ }^{1}$ & 2.97 & 42.8 & 8.75 & -1159.2 \\
$\mathrm{H}_{2}{ }^{2}$ & 3.23 & 32.19 & 9.95 & -1052.4 \\
${ }^{4} \mathrm{He}^{3}$ & 2.74 & 16.2 & 7.19 & -353.6 \\
${ }^{4} \mathrm{He}^{2}$ & 2.98 & 16.9 & 8.48 & -456.9 \\
\hline
\end{tabular}

TABLE I: Numerical values of $E_{\min }$ and $R_{\min }$, defined in the text. The parameters of the potential used in each case are from Refs. ${ }^{1}[53],{ }^{2}[54]$ and ${ }^{3}[34]$

[1] S. G. Brush, Statistical physics and the atomic theory of matter (Princeton U. P., 1983); See popular lecture on BEC by T.J. Greytak at this site: http://vmsstreamer1.fnal.gov/VMS_site_02/Lectures/NOS2002/Greytak/vf001.htm.

[2] F. London, Nature 141, 643 (1938).

[3] R.T. Azuah, W.G. Stirling,H.R. Glyde, M. Boninsegni, P.E. Sokol, S.M. Bennington, Phys. Rev. B56 ,14620 (1997).

[4] D.M. Ceperley, Rev. Mod. Phys. 67, 279 (1995).

[5] E.A. Cornell, C.E. Wieman, Nobel Lectures in Physics 2001, Rev. Mod. Phys., 74875 (2002).

[6] T. J.Greytak, D. Kleppner, D.G.Fried, T.C.Killian, L.Willmann, D.Landhuis and S.C.Moss, Physica B 280, 20 (2000).

[7] S. A. Moskalenko and D. W. Snoke, Bose-Einstein Condensation of Excitons and Biexcitons and Coherent Nonlinear Optics with Excitons (Cambridge U.P., 2000).

[8] V. Ginzburg and A. Sobyanin, JETP Lett. 15, 242 (1972).

[9] S.G Grebenev, B. Sartakov, J. P. Toennies and A. F. Vilesov, Science 289, 1532 (2000).

[10] A.Griffin, D.W.Snoke and S. Stringari, Bose-Einstein Condensation (Cambridge U.P., 1995).

[11] See especially the introductory article by D. W. Snoke and G. Baym in Ref. 10.

[12] M.C.Gordillo and D. M. Ceperley, Phys. Rev. Lett.79, 3010 (1997).

[13] M.C.Gordillo and D. M. Ceperley, Phys. Rev. B 65, 174527 (2002).

[14] Y. Kwon and K. B. Whaley, Phys. Rev. Lett. 89, 273401 (2002).

[15] H.J. Maris, G. M. Seidel and T. E. Huber, J. Low Temp. Phys. 51, 471 (1983); H.J. Maris, G.M. Seidel, F.I.B. Williams, Phys. Rev. B 36, 6799 (1987). 
[16] O.E. Vilches, J Low Temp. Phys. 89, 267 (1992).

[17] F.Ancilotto, M.M. Calbi, M.W. Cole, S.M. Gatica and E.S. Hernández, to appear in Israel J. Chem.; http://lanl.arXiv.org/abs/cond- mat/0307748.

[18] F. Ancilotto, M. M. Calbi, S. M. Gatica and M. W. Cole, submitted to Phys. Rev. Lett., http//lanl.arXiv.org/abs/cond-mat/030645019.

[19] G. Vidali, G. Ihm, H. Y. Kim and M. W. Cole, Surf. Sci. Rep. 12, 133 (1991).

[20] M. Muris, N. Dupont-Pavlovsky, M. Bienfait, and P. Zeppenfeld, Surf. Sci. 492, 67 (2001); J. C. Lasjaunias, K. Biljakovic, J. L. Sauvajol and P. Monceau, Phys. Rev. Lett. 91 , 025901 (2003).

[21] A. D. Migone and S. Talapatra,Gas Adsorption on Carbon Nanotubes" in the Encyclopedia of Nanoscience and Nanotechnology, published by American Scientific Publishers, editor H. S. Nalwa; Talapatra S, Rawat DS, Migone AD, J. of Nanoscience and Nanotechnology 2, 467 (2002); T. Wilson, O.E. Vilches, Phys. B 329, 278 (2003); M. Muris, M. Bienfait, P. Zeppenfeld, N. Dupont-Pavlovsky, M. Johnson, O.E. Vilches, T. Wilson, Appl. Phys. A 74, S1293 Part 2 Suppl. S (2002).

[22] T. Wilson, A. Tyburski, M.R. DePies, O.E. Vilches, D. Becquet, and M. Bienfait, J. Low Temp. Phys. 126, 403 (2002).

[23] Y.H. Kahng, R.B. Hallock, E. Dujardin, and T.W. Ebbesen, J. Low Temp. Phys. 126, 223 (2002); R.B. Hallock, L. Low Temp. Phys., in press.

[24] M. M.Calbi, S. M. Gatica, M. J. Bojan, G. Stan and M. W. Cole, Rev. Mod. Phys. 73, 857 (2001).

[25] B.K. Pradhan, G.U. Sumanasekera, K.W. Adu, H.E. Romero, K.A. Williams, P.C. Eklund Phys. B 323 (2002).

[26] A. C. Dillon, M.J. Heben, Appl. Phys. A, 72, 133 (2001).

[27] V.V. Simonyan, J.K. Johnson, J. of Alloys and Compounds 330, 659 (2002).

[28] S.R. Challa, D.S. Sholl, J.K. Johnson, J. Chem. Phys. 116, 814 (2002).

[29] R. A. Trasca, M.K. Kostov and M.W.Cole, Phys. Rev. B 67, 035410 (2003); M.C. Gordillo, J. Boronat and J. Casulleras, Phys. Rev. B 65, 014503 (2002).

[30] W. Shi and J. K. Johnson, Phys. Rev. Lett. 91, 015504 (2003).

[31] M. W. Cole, V. H. Crespi, G. Stan, C. Ebner, J. M. Hartman, S. Moroni, and M. Boninsegni, Phys. Rev. Lett. 84, 3883 (2000). 
[32] M. Boninsegni, S.Y. Lee, V.H. Crespi, Phys. Rev. Lett. 86, 3360 (2001).

[33] M. M. Calbi, F. Toigo and M. W. Cole, J. Low Temp. Phys. 126, 179 (2002).

[34] G. Stan and M.W. Cole, Surf. Science 395, 280 (1998).

[35] A.M. Rao, E. Richter, S. Bandow, B. Chase, P.C. Eklund, K.A. Williams, S. Fang , K.R. Subbaswamy, M. Menon, A. Thess, R.E. Smalley, G. Dresselhaus, M.S. Dresselhaus, Science 275, 187 (1997); S.M. Bachilo, M. S. Strano, C. Kittrell, R. H. Hauge, R. E. Smalley, and R. B. Weisman, Science 298, 2361 (2002).

[36] J.W.G. Wildoer, L.C. Venema, A.G. Rinzler, R.E. Smalley, C. Dekker, Nature 391, 59 (1998).

[37] A. Mizel, L. X. Benedict, M. L. Cohen, S. G. Louie, A. Zettl, N. K. Budraa, and W. P. Beyermann Phys. Rev. B 60, 3264 (1999).

[38] L. Henrard, E. Hernandez, P. Bernier, and A. Rubio Phys. Rev. B 60, R8521 (1999).

[39] Precisely the same mathematics determines the phonon density of states near a van Hove singularity; see J. M.Ziman, Principles of the Theory of Solids (Cambridge U.P., 1972), Sect. 2.5 .

[40] An example of such behavior occurs in the resonanting valence band model of superconductivity; see P.W. Anderson and Z. Zou, Phys. Rev. Lett. 60, 132 (1988).

[41] M.K.Kostov, J.C.Lewis and M.W.Cole, in Condensed Matter Theories, Vol. 16, edited by E.S. Hernández and J. Clark, Nova Science Publishers, NY,2001, p.161; an analogous study of elastic mediation of the interaction on a planar surface is discussed by K. H.Lau and W. Kohn, Surf. Sci. 65, 607 (1977) and 75, 69 (1978).

[42] S. M. Gatica, M. M. Calbi and M. W. Cole, to be published in J. Low Temp. Phys., cond-mat/0306614.

[43] L. W. Bruch, in Phase Transitions in Surface Films 2, ed. H. Taub, G. Torzo, H. J. Lauter and S. C. Fain, Jr. (Plenum, NY, 1991), p. 67.

[44] D.G.Narehood, J.V. Pearce, P.C. Eklund, P.E. Sokol, R.E. Lechner, J. Pieper, J.R.D. Copley, J. Cook, Phys. Rev. B 67, 205409 (2003).

[45] A.I. Skoulidas, D.M. Ackerman, J.K. Johnson, D.S. Sholl, Phys. Rev. Lett. 89, 185901 (2002).

[46] To quote G.V. Chester, Phys. Rev. A2, 256 (1970), "Little is known about the connection between Bose Einstein Condensation and Superfluidity". A contrasting opinion is presented by A. Griffin, in "Excitations in a Bose-condensed liquid", Cambridge University Press, 1993, p.16: "the changing properties as the temperature increases through $T_{\lambda}$ [are] direct conse- 
quences of the condensate ....vanishing at $T_{\lambda} "$.

[47] J. M. Kosterlitz and D. J. Thouless, in Progress in Low Temp. Phys., Vol. 7B, p. 371 (N. Holland, NY, 1978). The low $T$ phase is a superfluid with a power law decay of the order parameter.

[48] R. K. Pathria, Statistical Physics (Pergamon, Oxford, 1972), Sect. 7.5.

[49] M.M. Calbi, S.M. Gatica, M.J. Bojan and M.W, Cole, J. Chem. Phys. 115, 9975 (2001); A. Siber, Phys. Rev. B 66, 205406 and 235414 (2002).

[50] P. Schiffer et al, Phys. Rev. Lett. 74, 2379 (1995).

[51] A.P.Y.Wong and M.H.W.Chan, Phys.Rev. Lett. 65, 2567 (1990); D.S.Fisher, G. M. Grinstein and A. Khurana, Physics Today, 41, p. 58 (1988); R.J. Birgeneau et al, Phys. Rev. Lett. 54, 2147 (1985).

[52] P. W. Anderson, B. I. Halperin, and C. M. Varma, Phil. Mag. 25, 1 (1972); W. A. Phillips, J. Low Temp. Phys. 7, 351 (1972); C. E. Campbell, J. G. Dash and M. Schick,Phys. Rev. Lett. 26, 966 (1971).

[53] S.C. Wang, L. Senbetu and C.-W. Woo, J. of Low Temp. Phys. 41, 611 (1980).

[54] G. Stan, M.J. Bojan, S. Curtarolo, S.M. Gatica, M.W. Cole, Phys. Rev. B 62, 2173 (2000)) 


\section{FIGURE CAPTIONS}

1. Contours of the ground state transverse energy $E_{t}(\mathbf{R})-E_{\min }$ (relative to its minimum value) of an $\mathrm{H}_{2}$ molecule as a function of the radii $\mathbf{R}=\left(R_{1}, R_{2}, R_{3}\right)$ of the tubes bordering

the IC. $\mathbf{R}$ is measured relative to the minimum $\mathbf{R}_{\text {min }}$; the figure depicts behavior along the line $(0,1,-1)$. Beginning with the contour closest to the minimum (the closed curve), the contours correspond to relative energy 0.005, 0.01, 0.05, 0.1, 0.5, 1 and $5 \mathrm{~K}$.

2. Upper panel: transverse density of states $g(E)$ for ${ }^{4} \mathrm{He}$, computed from the distribution of ICs as described in the text. Lower panel: total density of states $N(E)$. In both cases, the energy is measured relative to the minimum $E_{\text {min }}=-456.88 \mathrm{~K}$

3. Transverse and total densities of states, as in Fig.2, for $\mathrm{H}_{2}$. $E_{\min }=-1052.97 \mathrm{~K}$

4. Specific heat of $\mathrm{He}$ (full curve) and $\mathrm{H}_{2}$ (dots) as a function of $T$ computed using the classical Boltzmann approach.

5. Fugacity $Z=\exp (\beta \mu)$ as a function of $T / T_{c}$ for two different densities: $1 \times 10^{-5} \AA^{-3}$ (full curve) and $0.5 \times 10^{-5} \AA^{-3}$ (dots) within an inhomogeneous nanotube array while the dashed curve corresponds to an ideal 4D gas having the same low energy density of states.

6. Condensate fraction as a function of the temperature computed for $\mathrm{H}_{2}$ in the nanotube system (full curve) and the $4 \mathrm{D} \mathrm{H}_{2}$ gas (dots) for $N=1 \times 10^{-5} \AA^{-3}$.

7. Heat capacity for $\mathrm{H}_{2}$ as a function of the temperature in nanotubes (full curve) and for the $4 \mathrm{D} \mathrm{H}_{2}$ gas (dashed) (panel a) and for ${ }^{4} \mathrm{He}$ in nanotubes (full curve) and for the $4 \mathrm{D}$ ${ }^{4}$ He gas (dashed) (panel b).

8. Density of $\mathrm{H}_{2}$ molecules (full curve) and ${ }^{4} \mathrm{He}$ atoms (dotted curve) as a function of transition temperature $T_{c}$. The short-dash $\left(\mathrm{H}_{2}\right)$ and long dash $(\mathrm{He})$ curves indicate the $4 \mathrm{D}$ ideal gas limiting behavior.

9. The occupied state energy distribution $n(E, T)$ (relative to the total number $N_{p}$ ) defined in Eq. 15 is shown for $\mathrm{H}_{2}$ at relative temperatures $T / T_{c}=0.5$ (full curve), 1 (dots), 1.1 (short dashes) and 1.5( long dashes). The integral of the curve at 0.5 is the fraction (about 0.7) of excited particles while the other curves integrate to 1 , since then the condensate fraction vanishes. 


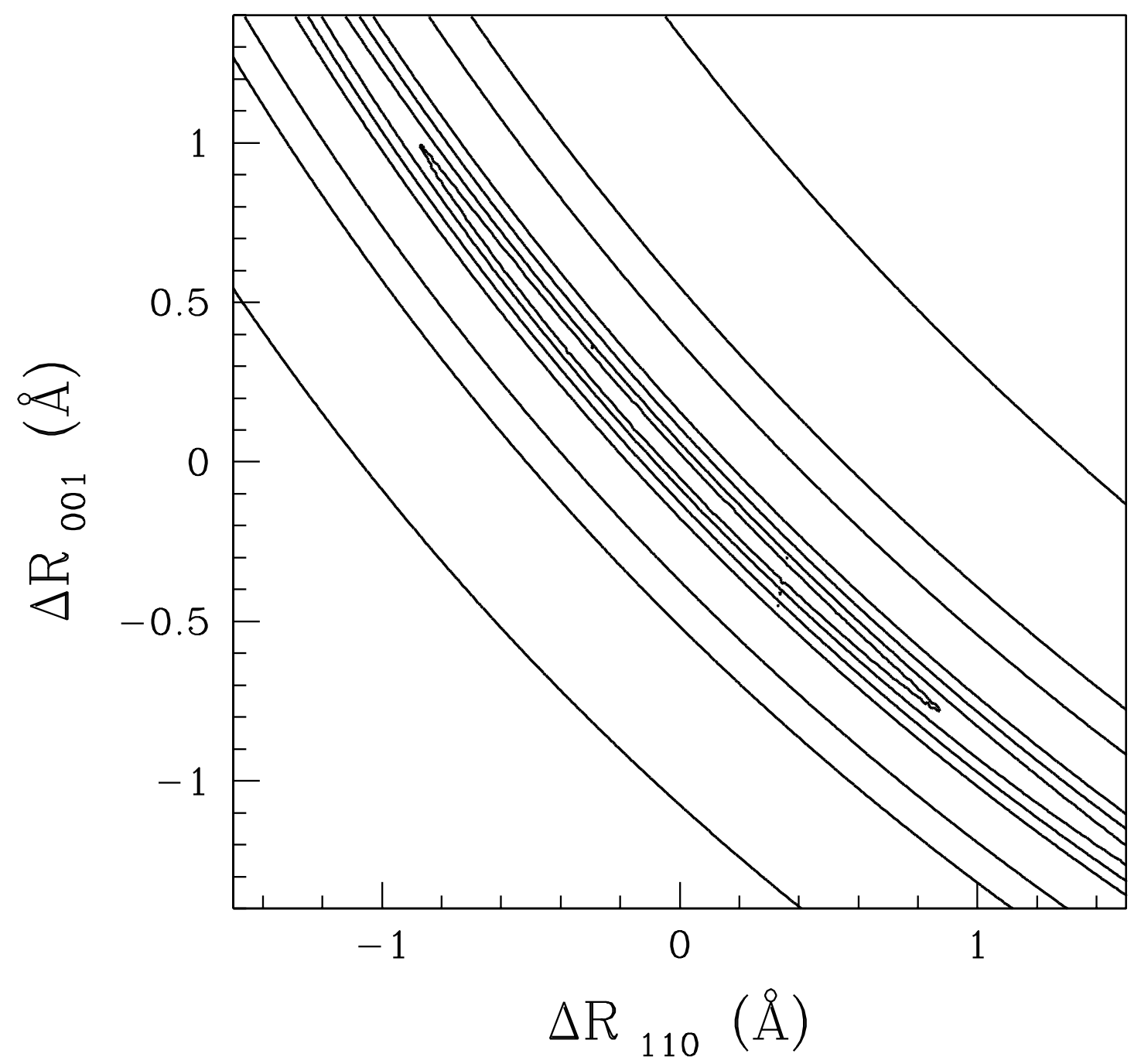



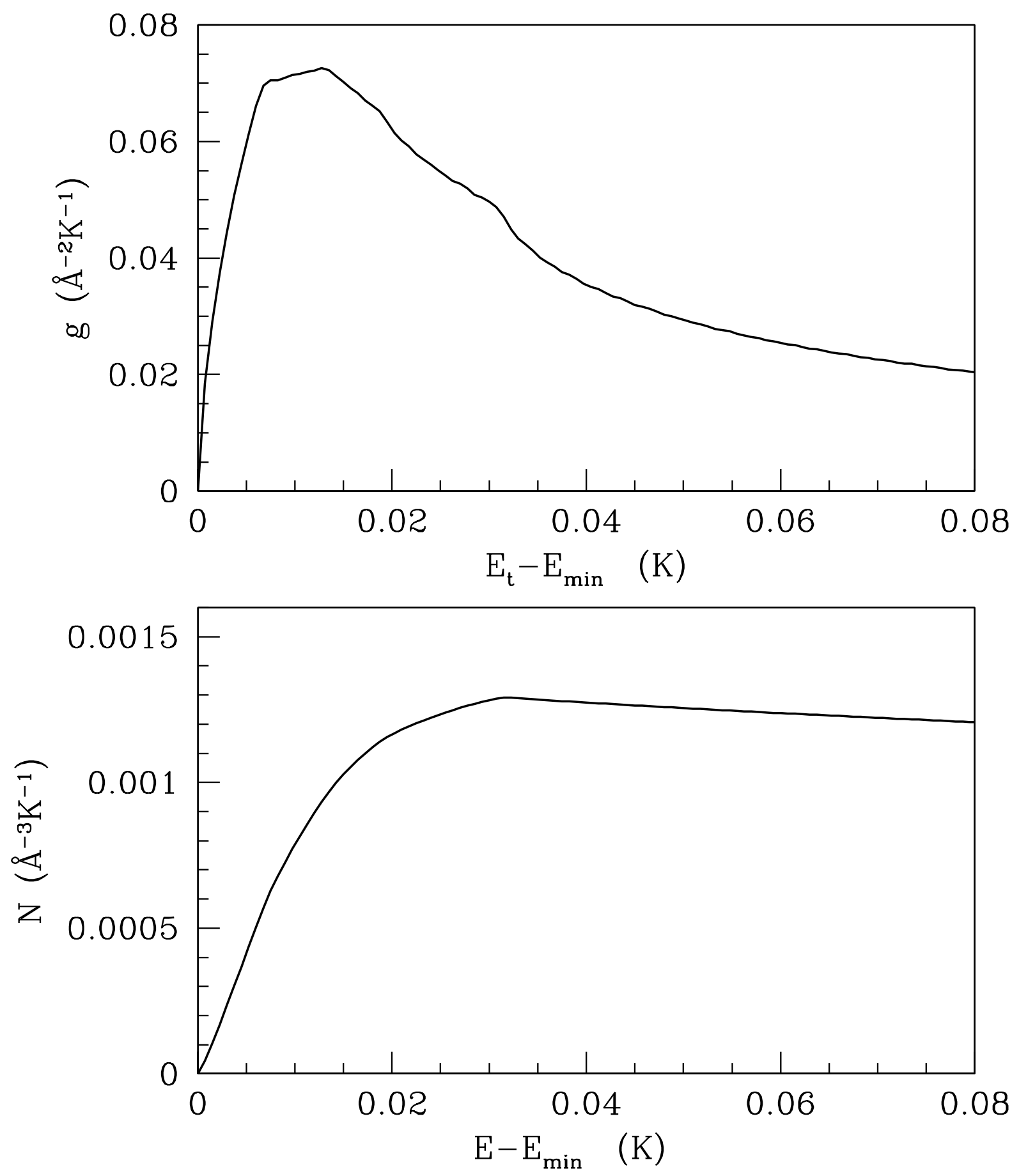

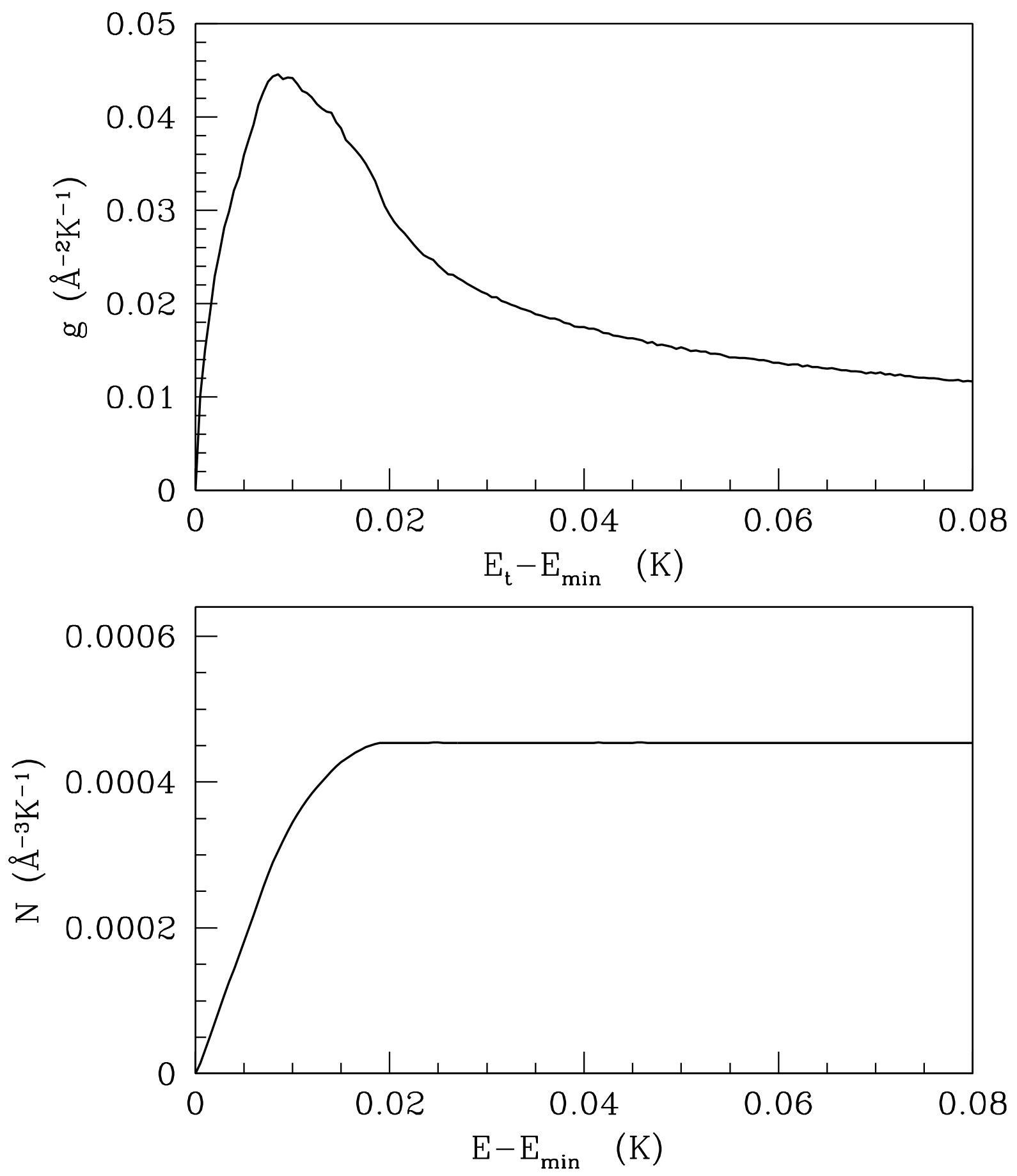


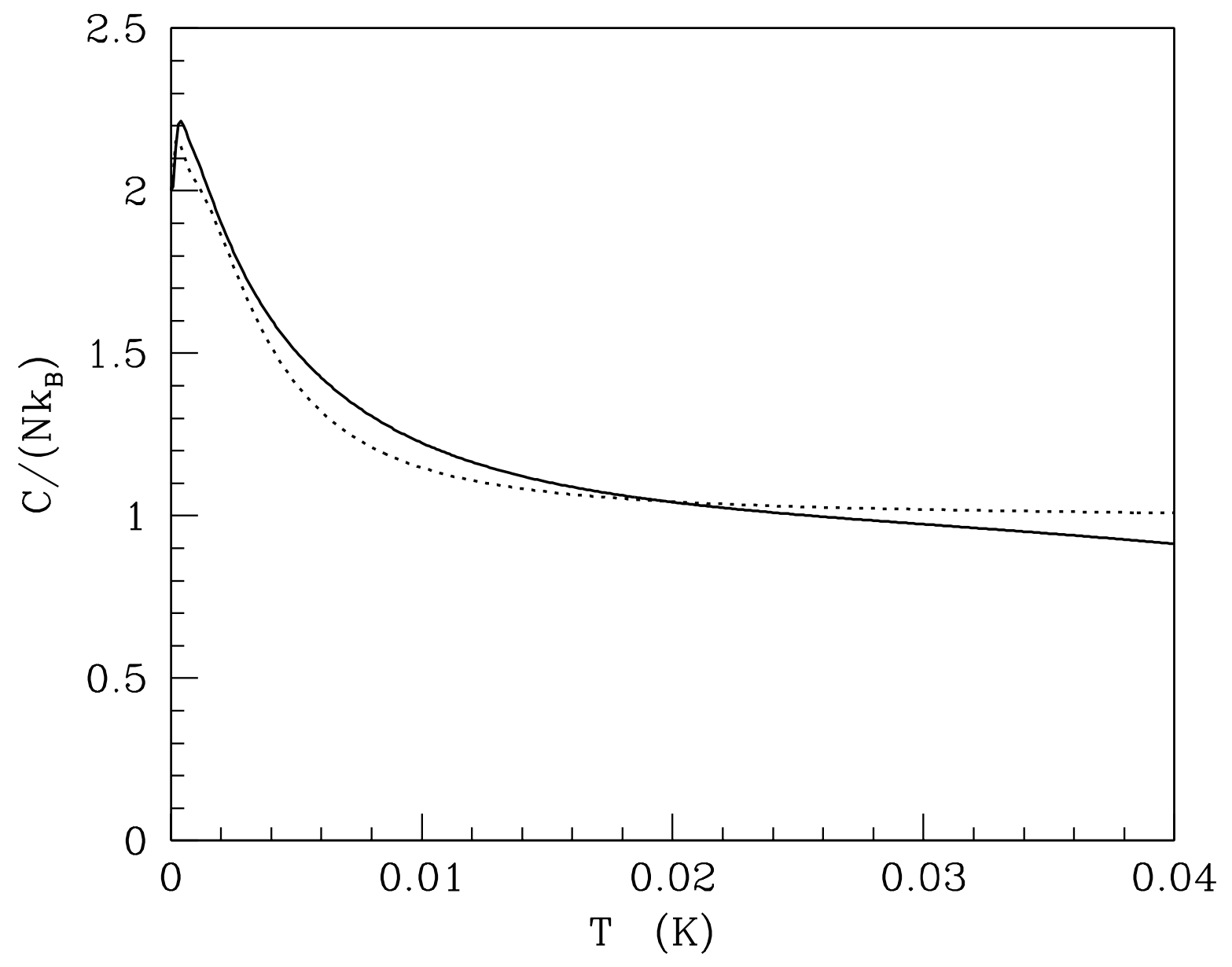




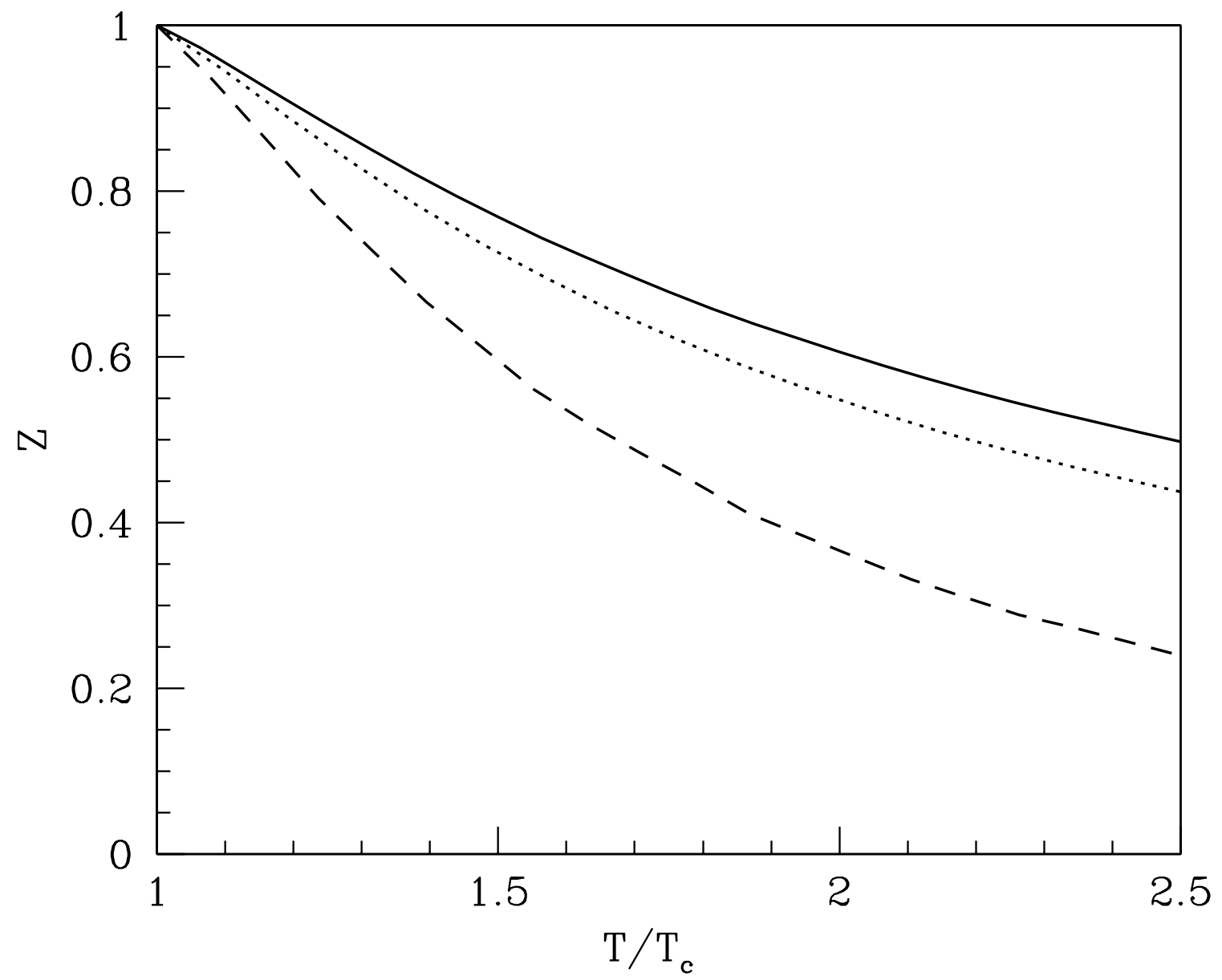




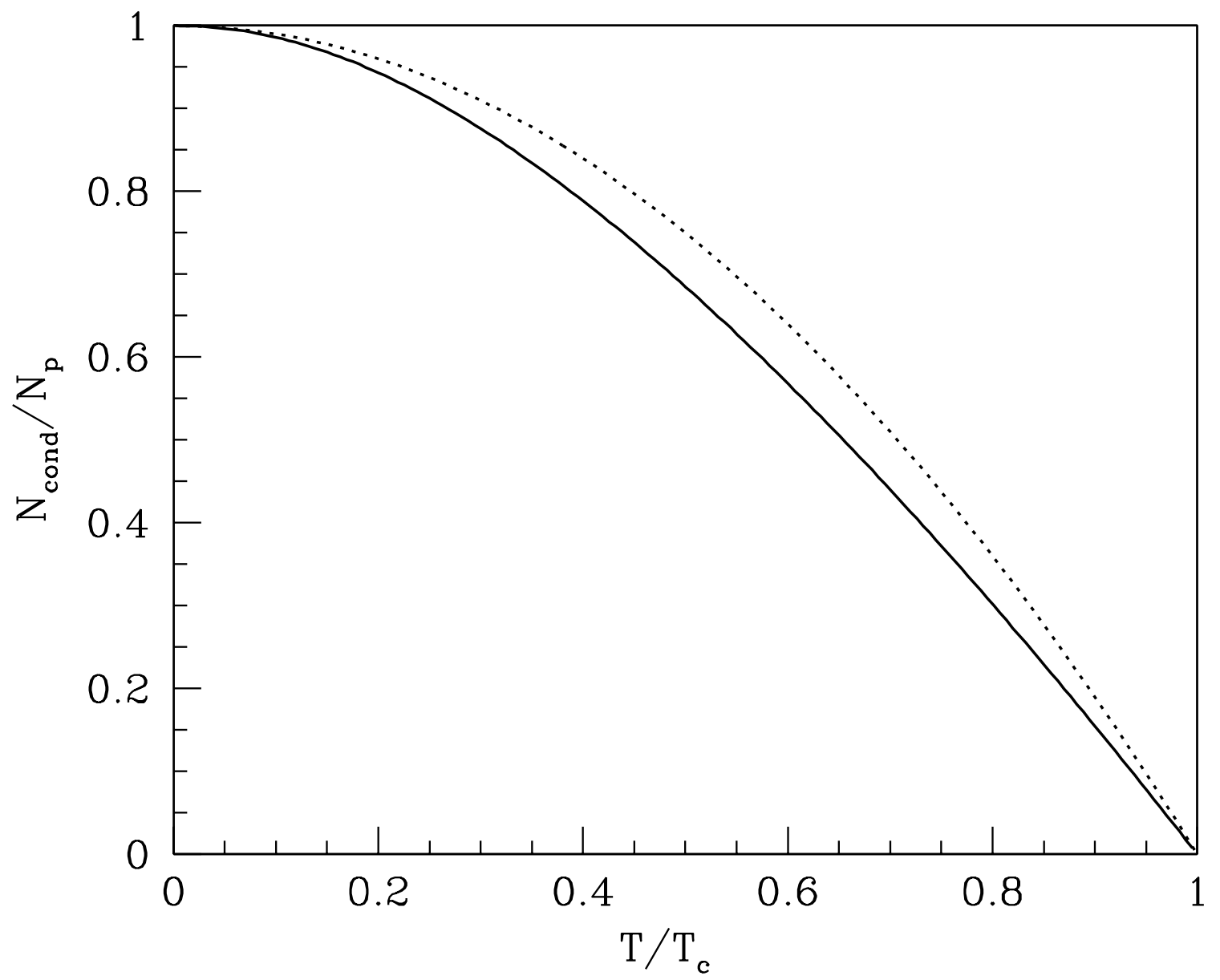




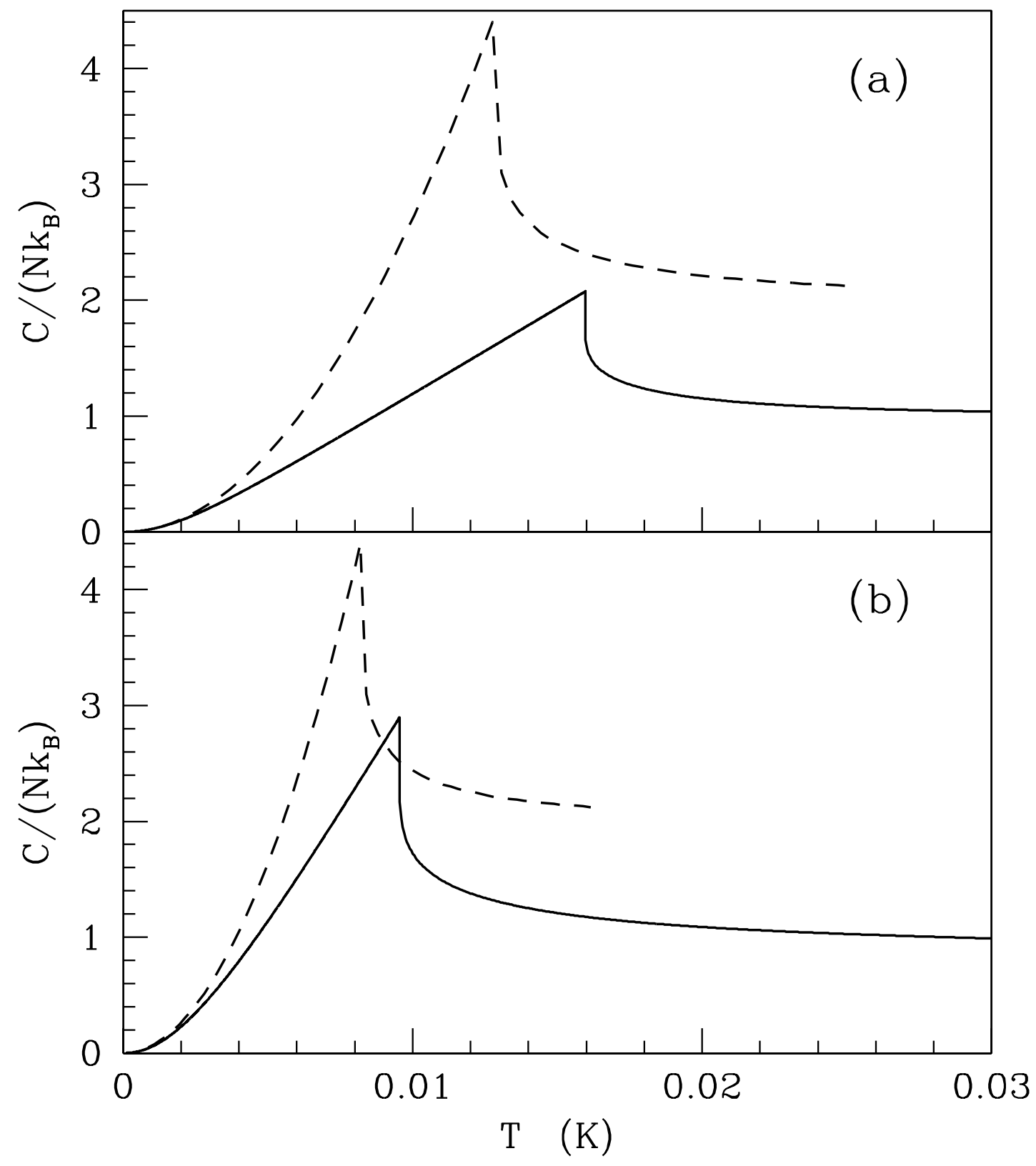




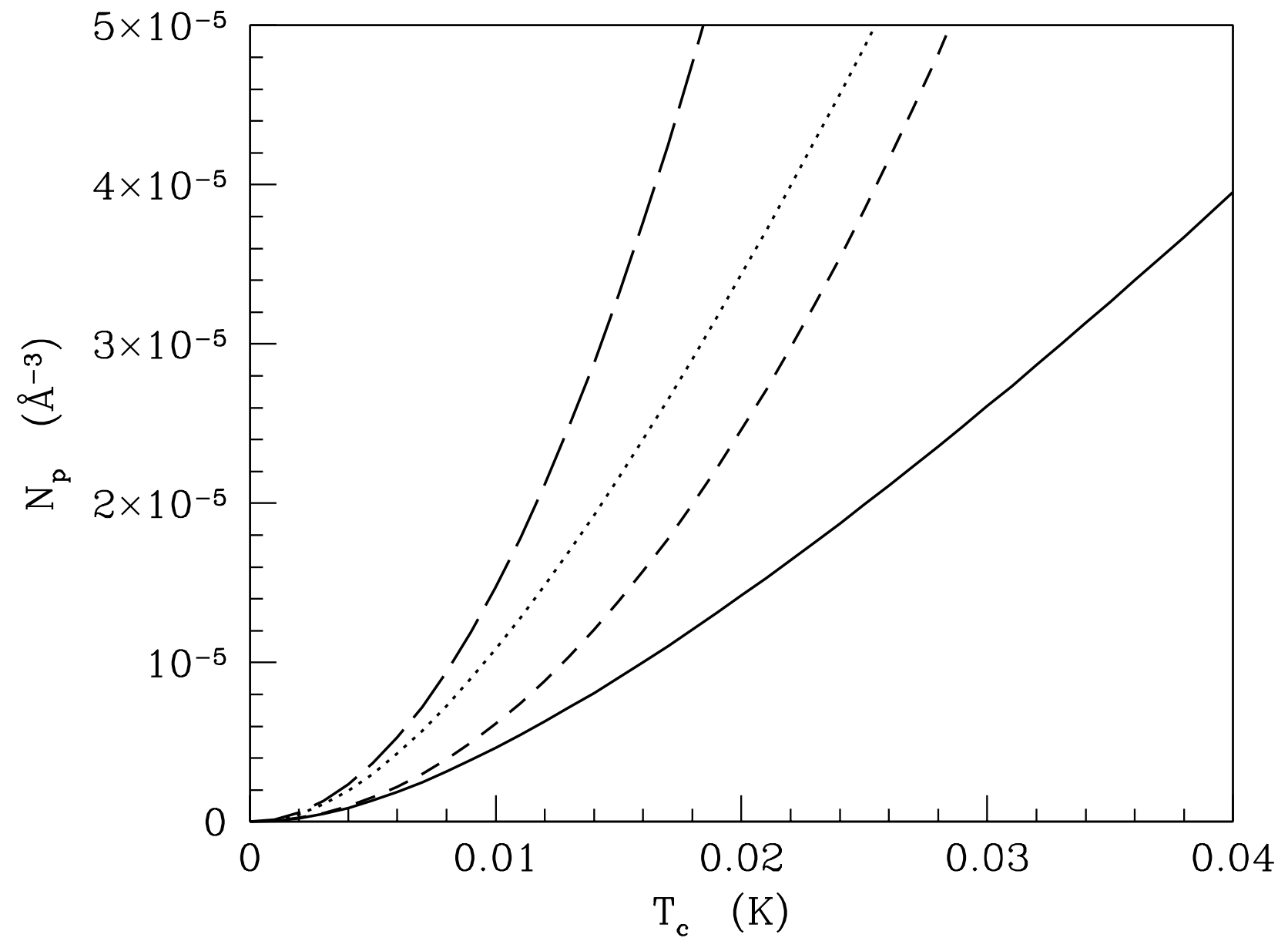




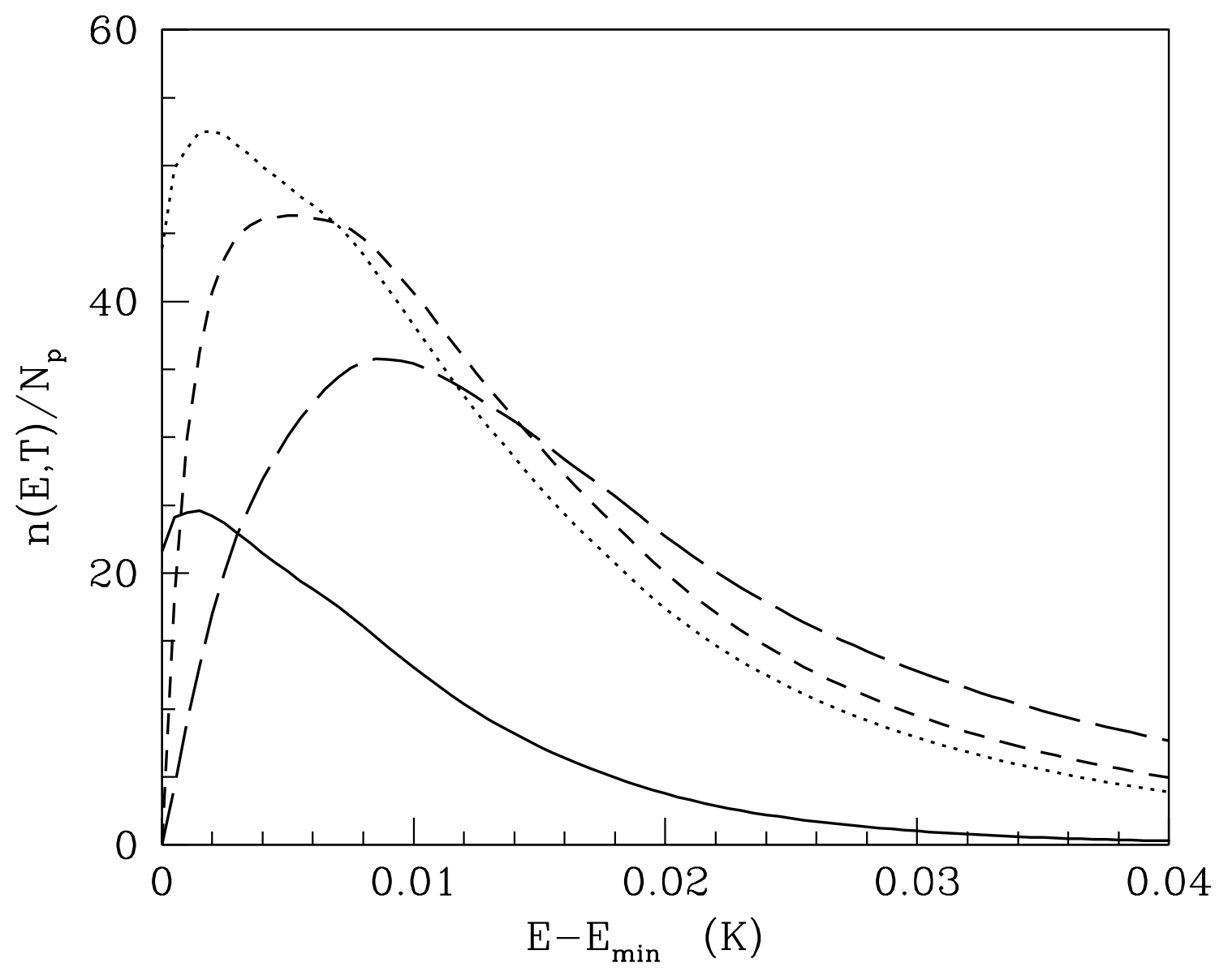

